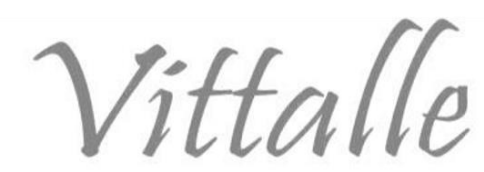

\title{
Fisiopatologia da COVID-19 e alvo farmacológico tromboimunológico
}

\author{
Maria Fernanda Grossi Hickmann ${ }^{\mathrm{a}}$, Rafael Costa Vieira Alexandre ${ }^{\mathrm{a}}$, Rafaela Oliveira \\ Gallart Morra $^{\mathrm{a}}$, Tayane Vasconcellos Pereira ${ }^{\mathrm{a}}$, Shana Priscila Coutinho Barroso ${ }^{\mathrm{b}}$, \\ Miguel de Lemos Neto ${ }^{c}$, Pedro Celso Braga Alexandre ${ }^{a^{*}}$
}

\author{
a'Universidade Federal do Estado do Rio de Janeiro - UNIRIO, Rio de Janeiro, RJ, Brasil \\ bLaboratório de Biologia Molecular, Instituto de Pesquisas Biomédicas, Hospital Naval Marcílio Dias, Marinha do \\ Brasil (IPB/HNMD/MB), Rio de Janeiro, RJ, Brasil
}

'Universidade Federal do Rio de Janeiro - UFRJ e Universidade do Estado do Rio de Janeiro - UERJ, Rio de Janeiro, RJ, Brasil

Histórico do Artigo:

Recebido em:

12/09/2020

Aceito em:

$28 / 10 / 2020$

Palavras-chave:

COVID-19;

fisiopatologia; drogas antitrombóticas

Keywords:

COVID-19;

pathophysiology;

antithrombotic drugs

\begin{abstract}
RESUMO
A pandemia da COVID 19 (coronavirus disease 2019) causada pelo coronavírus da síndrome respiratória aguda grave 2 (SARS-CoV-2) apresenta um desafio sem precedentes para identificação de drogas que possam prevenir e tratar a doença. Dado o rápido ritmo de descobertas científicas e dados clínicos gerados pelo grande número de pessoas rapidamente infectadas pelo novo vírus, os médicos precisam de evidências precisas sobre tratamentos médicos eficazes para essa infecção. A fisiopatologia é marcada por uma hiperinflamação responsiva à infecção viral, que é deflagrada pela "tempestade de citocinas", associada a um estado de hipercoagulação. Heparina de baixo peso molecular e heparina não fracionada são drogas que podem apresentar efetividade no tratamento. A presente revisão tem por objetivo evidenciar os achados clínicos que demonstrem a eficácia da terapia com heparina no tratamento da COVID-19. Até o presente momento muitos fármacos são utilizados em terapia combinada e a rápida expansão de conhecimento sobre a virologia do SARS-CoV-2 gera um direcionamento mais apurado na terapêutica. A pandemia de COVID-19 representa uma enorme crise de saúde pública mundial, e a velocidade e volume de ensaios clínicos buscam investigar terapias potenciais contra a infecção elucidando a capacidade e a necessidade de produzir evidências de alta qualidade durante a pandemia.
\end{abstract}

Physiopathology of COVID-19 and thromboimmunological pharmacological target

\section{ABSTRACT}

The pandemic of coronavirus disease 2019 (COVID-19) caused by the novel severe acute respiratory syndrome coronavirus 2 (SARS-CoV-2) presents an unprecedented challenge to identify effective drugs for prevention and treatment. Given the rapid pace of scientific discovery and clinical data generated by the large number of people rapidly infected by new virus, clinicians need accurate evidence regarding effective medical treatments for this infection. Pathophysiology is marked by hyperinflammation responsive to viral infection, which is triggered by the "cytokine storm", associated with a state of hypercoagulation. Low molecular weight heparin and unfractionated heparin are drugs that can be effective in the treatment. The present review aims to highlight the clinical findings that demonstrate the effectiveness of Heparin therapy in the treatment of COVID-19. To date, many drugs are used in combination therapy and the rapid expansion of knowledge about the virology of SARS-CoV-2 generates a more accurate direction in therapy. The COVID-19 pandemic represents a huge public health crisis worldwide, and the speed and volume of clinical trials seek to investigate potential therapies against infection by elucidating the ability and need to produce high-quality evidence during the pandemic.

\section{Introdução}

A pandemia da COVID-19 (Corona Virus Disease 2019), causada pelo coronavírus da síndrome respiratória aguda grave 2 (SARS-CoV-2) começou em Wuhan, China, em

\footnotetext{
* Autor correspondente: pedro.alexandre@unirio.br (Alexandre P.C.B.)
} 
dezembro de 2019, e desde então se espalhou rapidamente pelo mundo (1). O SARSCoV-2 pertence ao grupo dos $\beta$-coronavírus, assim como o SARS-CoV - causador da síndrome respiratória aguda grave e o MERS-COV responsável pela síndrome respiratória do Oriente Médio (2). Essa família viral possui características estruturais em comum, como a presença de envelope e nucleocapsídeo, o qual é composto por RNA fita simples com sentido positivo. O nucleocapsídeo é revestido por uma bicamada fosfolipídica, a qual apresenta-se envolta por proteínas de membrana, além de duas glicoproteínas 'spike': a proteína 'spike' $\mathrm{S}$, existente em todos os $\beta$-coronavírus, e a hemaglutinina-esterase, presente em apenas alguns vírus do grupo (3).

A partir da infecção, o paciente pode apresentar sintomas como febre $(87,9 \%)$, fadiga $(38,1 \%)$, tosse $(67,7 \%)$, dispneia $(18,6 \%)$ e mialgia $(14,8 \%)$, com a possibilidade de evolução para pneumonia, síndrome respiratória aguda grave, insuficiência cardíaca, sepse, falência renal aguda e, por fỉm, óbito (4). A progressão da doença envolve a ativação de uma cascata de citocinas inflamatórias e eventos pró-trombóticos. Estes eventos estão relacionados a numerosos fatores de risco, como hipertensão, câncer, idade avançada, doença cardiovascular prévia, diabetes e doença respiratória crônica (5).

Esta revisão resume evidências atuais sobre a virologia, fisiopatologia e uso de heparina como tratamento antitrombótico propostos na intervenção terapêutica da COVID-19.

\section{Método}

Este trabalho é uma revisão de literatura com abordagem qualitativa, método descritivo-reflexivo, com base nos referenciais bibliográficos publicados em formato de artigo em revistas indexadas, disponíveis nas bases de dados: PubMed; Scielo, LILACS e Google Acadêmico.

As buscas foram realizadas utilizando os termos: coronavírus, síndrome respiratória aguda grave e coronavírus 2; 2019-nCoV, SARS-CoV-2, SARS-CoV, MERS-CoV, COVID-19, SARS-CoV e fisiopatologia, SARS-CoV e dímero-D, SARS-CoV e tromboembolismo, SARS-CoV e heparinas em estudos publicados de $1^{\circ}$ de janeiro de 2020 a $1^{\circ}$ de setembro de 2020.

\section{Fisiopatologia}

Em relação à fisiopatologia da COVID-19, o vírus SARS-CoV-2 pode atravessar a membrana das mucosas, especialmente laríngea e nasal (10) a partir da aspiração de gotículas exaladas por pacientes contaminados, ou seja, contendo o vírus (11), que entra nos pulmões através do trato respiratório (10). O vírus utiliza o receptor da Enzima Conversora da Angiotensina 2 (ECA-2) para adentrar as células. Esse receptor é expresso nos cardiomiócitos, pneumócitos tipo 2, epitélio renal e gastrointestinal (7-9), exercendo importante papel protetor em órgãos vitais a partir da regulação negativa do sistema renina angiotensina (SRA) $(6,8)$. A ligação viral ao ECA-2 ocorre por meio da proteína spike $S$. Essa ligação causa alterações conformacionais na proteína spike $S$, garantindo a fusão do envelope viral na membrana celular (9) e promovendo a endocitose do material genético viral (6-9).

A fisiopatologia da COVID-19 se assemelha fortemente à exibida em resposta a infecção pelo SARS-CoV (9). Ambos os vírus promovem a regulação negativa do receptor ECA-2. A partir desse mecanismo, a regulação do SRA é prejudicada, favorecendo a lesão de órgãos vitais como pulmão, coração e rins (8).

Associado a este efeito sobre o SRA, há uma importante resposta imune com intensa 
produção de citocinas pró-inflamatórias, configurando a chamada "tempestade de citocinas". Destaca-se a presença da interleucina 6 (IL-6) $(6,9)$, produzida principalmente por macrófagos e leucócitos ativados, e responsável por ativar linfócitos $\mathrm{B}$ e $\mathrm{T}$, além de induzir a produção de proteínas de fase aguda, como a proteína $\mathrm{C}$ reativa (12). Essa "tempestade de citocinas" tem participação fundamental no desfecho do caso, devido à forte injúria a órgãos vitais por ela promovida, podendo causar falência múltipla dos órgãos (9), além de sepse, lesão miocárdica e vascular (7). A hiperreatividade imune ao vírus é essencialmente importante no universo pediátrico, visto que a tempestade de citocinas pode resultar na Síndrome Inflamatória Multissistêmica (SIM). Descrita em recentes relatórios provenientes da Europa e América do Norte, a SIM se apresenta similar a doença de Kawasaki, podendo evoluir a falência múltipla de órgão e choque tóxico (18).

A partir da replicação inicial do SARS-CoV-2, sucedida nos pneumócitos tipo II (23, 24), os danos ao parênquima pulmonar se tornam aparentes. A injúria inicial acarreta uma resposta imune local, com vasodilatação, recrutamento de leucócitos e aumento da permeabilidade vascular $(15,16)$, caracterizado por hiperplasia reativa dos pneumócitos, presença de exsudato e infiltrado inflamatório multinucleado (6). Esse dano adicional causa hipoxemia e, consequentemente, lesão cardiovascular (15). O ataque ao sistema respiratório pode evoluir rapidamente para pneumonia e síndrome respiratória aguda grave, agravando o quadro hipoxêmico (6). Em estágio mais tardio da doença, com alta replicação viral, há um comprometimento da barreira epitelial-endotelial. Essas alterações na permeabilidade endotelial identificadas levam a instauração do vírus e ao caráter sistêmico da doença (24). Com isso, iniciam-se as manifestações extrapulmonares nos sítios cujos tecidos expressam o receptor ECA-2 (7-9).

Sabe-se que aproximadamente $7,5 \%$ das células cardíacas expressam o receptor ECA2, portanto é um órgão potencialmente afetado em casos de infecção pelo novo coronavírus $(6,7)$. Os mecanismos de lesão ainda não estão elucidados por parte da comunidade científica, embora sejam provavelmente multifatoriais (7). O SARS-CoV-2 pode causar miocardite viral e consequente resposta inflamatória (6). Estudos por meio de transcrição reversa seguida em reação em cadeia da polimerase (RT-PCR) mostram a partícula viral presente nos cardiomiócitos, sustentando a possibilidade de ocorrência de cardiotoxicidade direta (7). Juntamente aos danos isquêmicos provocados pela hipóxia tecidual, as injúrias cardíacas podem ser observadas através da detecção de níveis elevados de troponina e outros marcadores cardíacos, além de alterações no eletrocardiograma (ECG) e ecocardiograma (ECO) (6).

A hiper inflamação responsiva a infecção viral pode causar inflamação vascular com a exposição do fator tecidual $(13,14)$, instabilidade das placas de ateroma e ativação patológica da trombina, instaurando um estado de hipercoagulação $(6,13)$. Essa hipercoagulação pode dar origem a trombose venosa profunda (TVP) e tromboembolismo pulmonar (TEP) até mesmo a instalação da coagulação intravascular disseminada (CID) $(13,14,16)$. O biomarcador dímero-D, produto da degradação da fibrina, permite identificar a ocorrência de um estado de hipercoagulação $(13,16)$. É importante ressaltar que a elevação desse marcador é comum em pacientes críticos, sendo sinal de mau prognóstico da doença (6).

Dessa forma, as manifestações cardiovasculares da COVID-19 incluem a elevação de biomarcadores miocárdicos, arritmias, trombose venosa profunda, coagulação intravascular disseminada (13) e choque cardiogênico (6).

\section{Antitrombóticos}


Recentes estudos descreveram que a COVID-19 está relacionada a complicações de coagulopatia, e CID na maioria dos pacientes que morreram pela doença (18). De muitas maneiras a pandemia de COVID-19 afetou a prevenção e o manejo de doenças tromboembólicas e trombóticas. Primeiramente, por efeitos diretos e indiretos da infecção, como nos casos graves e hipóxia. Em segundo lugar pela resposta inflamatória sistêmica associada aos fatores de risco já conhecidos, tornam os pacientes mais predispostos a eventos trombóticos (19).

Estudo retrospectivo de 183 pacientes com pneumonia por COVID-19 confirmada no hospital de Tongji na China, mostrou que os que morreram $(11,5 \%)$ tiveram níveis mais altos de dímero D, aumento do tempo de protrombina (PT) e do tempo de tromboplastina parcial ativada na apresentação em comparação com aqueles que sobreviveram. Ficou evidente que parâmetros anormais de coagulação (PT prolongada e dímero D aumentado) são preditores de mau prognóstico e podem ser alvos terapêuticos importantes (20).

Heparina de baixo peso molecular e heparina não fracionada possuem propriedades antiinflamatórias por meio do bloqueio de selectina, da inibição de bradicinina, da geração de trombina e pela ligação com citocinas pró-inflamatórias (25). Heparinas também têm propriedades antivirais, como por exemplo, heparina pode atenuar interação viral de SARS-CoV-2 com receptor de ECA-2 (21). Esse efeito antiinflamatório também pode ser exercido pelos anticoagulantes diretos orais, como o inibidor do fator Xa (22). Em um estudo retrospectivo com 449 pacientes, no hospital de Tongji na China, 99 pacientes com quadro grave de COVID-19, que receberam tratamento com heparina de baixo peso molecular por sete dias, demonstraram melhor prognóstico do que os que não receberam (18).

\section{Conclusão}

A fisiopatologia da doença indica uma tendência a formação de trombos no estado de hiperinflamação pelo COVID-19 e a literatura atual sobre a utilização de antitrombóticos no COVID-19 vem mostrando eficácia no sentido de evitar a coagulação intravascular disseminada, além de ser utilizada nos protocolos dos principais centros de saúde. Sendo assim, os estudos atuais mostram a importância da utilização de agentes antitrombóticos nos protocolos de tratamento do novo coronavírus.

Por fim, pode-se afirmar que a observação dos resultados obtidos nos ensaios em andamento e novos estudos randomizados, com metodologias avançadas, são imprescindíveis para ratificar a eficácia dos agentes antitrombóticos no tratamento da COVID-19.

\section{Referências}

1. Sanders J, Monogue M, Jodlowski, T and Cutrell J., 2020. Pharmacologic Treatments for Coronavirus Disease 2019 (COVID-19). JAMA 2020; 323(18): 1824-1836.

2. Xu X, Ong Y, Wang D. Role of adjunctive treatment strategies in COVID-19 and a review of international and national clinical guidelines. Military Medical Research 2020; 7(1): 22.

3. Jin Y, Yang H, Ji W, Wu W, Chen S, Zhang W et al. Virology, Epidemiology, Pathogenesis, and Control of COVID-19. Viruses 2020; 12(4): 372.

4. Chen T, Wu D, Chen H, Yan W, Yang D, Chen G et al. Clinical characteristics of 113 deceased patients with coronavirus disease 2019: retrospective study. BMJ 2020; 368: m1091.

5. Jordan R, Adab P, Cheng K. Covid-19: risk factors for severe disease and death. BMJ 2020; 368: m1198. 
6. Geng Y, Wei Z, Qian H, Huang J, Lodato R, Castriotta R. Pathophysiological characteristics and therapeutic approaches for pulmonary injury and cardiovascular complications of coronavirus disease 2019. Cardiovascular Pathology 2020; 47: 107228.

7. Kang Y, Chen T, Mui D, Ferrari V, Jagasia D, Scherrer-Crosbie M et al. Cardiovascular manifestations and treatment considerations in COVID-19. Heart 2020; 106(15): 1132-1141.

8. Javanmard S, Heshmat-Ghahdarijani K, Vaseghi G. Angiotensin-converting-enzyme inhibitors (ACE inhibitors) and angiotensin II receptor blocker (ARB) use in COVID-19 prevention or treatment: A paradox. Infection Control \& Hospital Epidemiology 2020; 1-2.

9. Zoller M. Coronavirus disease 2019 [Internet]. https://link.springer.com/article/10.1007/s00101-02000761-2. 2020 Available from: https://link.springer.com/article/10.1007/s00101-020-00761-2

10. Lin L, Lu L, Cao W, Li T. Hypothesis for potential pathogenesis of SARS-CoV-2 infection-a review of immune changes in patients with viral pneumonia. Emerging Microbes \& Infections 2020; 9(1): 727-732.

11. Xu Y, Dong J, An W, Lv X, Yin X, Zhang J et al. Clinical and computed tomographic imaging features of novel coronavirus pneumonia caused by SARS-CoV-2. Journal of Infection 2020; 80(4): 394-400.

12. Schulte W, Bernhagen J, Bucala R. Cytokines in Sepsis: Potent Immunoregulators and Potential Therapeutic Targets-An Updated View. Mediators of Inflammation 2013; 2013:1-16.

13. Jara González F, Jimenez Alulima G, Sananay Auquilla E, Murillo Sanclemente J, Molina Vasquez P, Vélez Páez J. Hipercoagulabilidad, trombosis intravascular y trombocitosis asociada al COVID-19. Reporte de un caso. Bionatura 2020; 5(2): 1138-1141.

14. Vivas D, Roldán V, Esteve-Pastor M, Roldán I, Tello-Montoliu A, Ruiz-Nodar J et al. Recomendaciones sobre el tratamiento antitrombótico durante la pandemia COVID-19. Posicionamiento del Grupo de Trabajo de Trombosis Cardiovascular de la Sociedad Española de Cardiología. Revista Española de Cardiología 2020; 73(9): 749-757.

15. Su S, Wong G, Shi W, Liu J, Lai A, Zhou J et al. Epidemiology, Genetic Recombination, and Pathogenesis of Coronaviruses. Trends in Microbiology 2016; 24(6): 490-502.

16. Giannis D, Ziogas I, Gianni P. Coagulation disorders in coronavirus infected patients: COVID-19, SARS-CoV-1, MERS-CoV and lessons from the past. Journal of Clinical Virology 2020; 127: 104362.

17. Joseph, Tinku. COVID-19 E-Book. "International Pulmonologist's consensus on COVID-19" - 2nd Edition; 2020.

18. Tang N, Bai H, Chen X, Gong J, Li D, Sun Z. Anticoagulant treatment is associated with decreased mortality in severe coronavirus disease 2019 patients with coagulopathy. Journal of Thrombosis and Haemostasis 2020; 18(5): 1094-1099.

19. Bikdeli B, Madhavan M, Jimenez D, Chuich T, Dreyfus I, Driggin E et al. COVID-19 and Thrombotic or Thromboembolic Disease: Implications for Prevention, Antithrombotic Therapy, and Follow-Up. Journal of the American College of Cardiology 2020; 75(23): 2950-2973.

20. Arachchillage D, Laffan M. Abnormal coagulation parameters are associated with poor prognosis in patients with novel coronavirus pneumonia. Journal of Thrombosis and Haemostasis 2020; 18(5): 1233-1234.

21. Bikdeli B, Madhavan MV, Gupta A, Jimenez D, Burton JR, Der Nigoghossian C et al.; Global COVID-19 Thrombosis Collaborative Group. Pharmacological Agents Targeting Thromboinflammation in COVID-19: Review and Implications for Future Research. Thromb Haemost 2020; 120(7): 1004-1024.

22. Jose R, Manuel A. COVID-19 cytokine storm: the interplay between inflammation and coagulation. The Lancet Respiratory Medicine 2020; 8: e46-e47.

23. Yao X, He Z, Li T, Zhang H, Wang Y, Mou H et al. Pathological evidence for residual SARS-CoV-2 in pulmonary tissues of a ready-for-discharge patient. Cell Research 2020; 30(6): 541-543.

24. Wiersinga W, Rhodes A, Cheng A, Peacock S, Prescott H. Pathophysiology, Transmission, Diagnosis, and Treatment of Coronavirus Disease 2019 (COVID-19). JAMA 2020; 324(8):782.

25. Young E. The anti-inflammatory effects of heparin and related compounds. Thromb Res 2008; 122(6): 743-52. 